TRITA-EPP-82-04

\title{
PARADIGM TRANSITION IN COSMIC PLASMA PHYSICS
}

Hannes A. fuén

June 1982

Introductory lecture at the Conference on Plasma Physics in Gothenburg, June 1982 .

Department of Plasma Physics Royal Institute of Technology S-100 44 Stockholm, Sweden 
PARADIGM TRANSITION IN COSMIC PLASMA PHYSICS

H. Alfvén

Royal Institute of Technology, Department of Plasma Physics, s-100 44 stockholm, Sweden

\section{Abstract}

In situ measurements in the magnetospheres together with general advancement in plasma physics are now necessitating introduction of a number of effects that have been recently discovered or earlier neglected. Examples are:

Electric double layers (Iike in the lower magnetosphere)

Thin current layer (like in the magnetopause) giving mpace: a cellular structure.

Current produced filaments $(e . g$. in prominences, solar corona and interstellar clouds $\mathrm{s}$.

Further it is important to use the electric current (particle) déscription and to study the whole aircuit in which the current flows. The pinch effect cannot be neglected as is now usually done. The:critical velocjty phenomenon is essential, for example for the band structure of solar system. Theory of dusty plasmas is important.

The result is a change in so many theories in cosmic plasma physics that It is appropriate to speak of an introduction of a new paradigm. This should be based on empirical knowledge from magnetospheric and laboratory investigations. Its application to astrophysics in general, including cosmology. will necessarily lead to a revision of $\mathrm{e} . \mathrm{g}$. the present theories of the formation of stars, planets and satelittes. It is doubtful whether the big bang cosmology will survive. 
PARADIGM TRANSITION IN COSMIC PLASMA PHYSICS

Introductory lecture at the conference on Plasma Physics

in Gothenburg; June 1982 by Hannes Alfven

I Towards a unified theory of cosmic plasma physics 1

II New "discoveries"

A Double layers

B Energy transfer

AuroreI circuit

Iocal Plasma_properties_and the_circuit

Boundary conditions

Magrietic merging theories

Dualism in cosmic plasma physics

Magnetic field versus electric current_(particle)

description

D Application to solar wind - nagnetospheric interaction

Simple model

Approach to a more realistic_model

E Newtonian attraction and electromagnetic attraction 9

Filamentary structure of cosmic plasmas

The neglected pinch effect 10

$F$ Celiular structure of space 11

Current_layers in the magnetospineres $\quad 11$

Cell walls in the magnetospheres and in more

distant reqions

Space In space age

II Application of the new paradigm to cosmology and solar system evolution

A The new concept of space and the existence of antimatter

B Annihilation as a source of energy 13

Energy release in guasars 13

The Hubble expansion 14

C Approach to cosmology 15

Should the big bang hypothesis be considered_as

sacrosanct?

Confrontation between three-dingnsional highly structured model and four dimensionel homogeneous model 
D Evolution of interstellar clouds to stars,

planets and satelites

Dusty_plasma

Pinch_effect

The_critical_velocity

Transitition from_plasma to plantesimals and the_ $2: 3$ contraction 


\section{Towards a unified theory of cosmic plasma physics}

Cosmic plasma physics is at present in a state of revision which is so drastic that it is appropriate to speak of a change in paradigm. This change started 5 or 10 years ago, and it will probably take at least 10 years more until the transition is completed. The process is precipitated by:

(a) In situ measurements of the properties of plasmas in the magnetospheres (including the solar nagnetosphere = heliosphere = = solar wind region). Active space experiments are beginning to make significant contributions.

(b) Laboratory experiments. Cosmic piasma physics is profiting from thermonuclear research, but those experiments which are especially designed to clarify cosmic situations are of decisive importance.

(c) An increased understanding how to transfer knowledge from one plasma region to another.

The present paper will be based on the assumption that the basic properties of plasmas are the same everywhere.

This means that we are attempting to develop a unified theory of cosmic plasmas covering 27 orders of magnitude, from laboratory experiments (typically $0.1 \mathrm{~m}$ ) up to the Fubble distance $10^{26} \mathrm{~m}$. This large distance is covered in "a cosmic triple jump" (see Fig. 1). The first step of $10^{9}$ brings us from laboratory to magnetosphere (typically $\sim 10^{8} \mathrm{~m}$ ), next hop to intersteliar phenomena, typically $\sim 10^{17} \mathrm{~m}$ (=10 light years). The third jump of $10^{9}$ brings us up to the Hubble distance $\left(\sim 10^{26} \mathrm{~m}\right)$.

The laboratory and magnetosphere regions are accessible to in situ measurements lout to the region of the giant planets which is $\left.\sim 10^{12} \mathrm{~m}\right)$. In these regions it is possible to make high quality diagnostics. This means that - at least in principle - it is possible to develop theories of real scientific value. Outside the reach of spacecraft we cannot explore the plasma with the same degree of reliability, so all theories are necessarily somewhat speculative. This applies to solar and cometary physics and to the physics of galactic and extragalactic phenomena. It includes such important fields as the theory of the formation of 
stars, planets and satellites, and cosmology, also because some essential processes took place long ago.

From this follows that our method of approach should be:

(a) Dexive from laboratory and magnetopsheric measurements general laws governing cosmic plasmas.

(b) Apply such laws to solar, interstellar, galactic, and cosmological problems (C.P. I) *.

II New "discoveries"

The new paradigm derives from a number of "discoveries". Some of these refer to phenomena which have not been known earlier (e.g, magnetopause and double layers in magnetized plasmas). Some are well- known since long ago but have not until recently beenapplied to cosmic plasma physjcs (e.g. electric doubie layers and pinch effect). The most important "discoveries" will be summarized in this part II (and also in Part III).

A. Double layers

Since the time of Iangmuir it is known that electric double layers often occur in non-magnetized laboratory plasmas and recently it has been demonstrated that they also exist in magnetized plasmas (Fig. 2,3). Because of the poor contact between laboratory plasma physics and the physics of space plasmas, it is not unil quite recently that double layers in cosmic piasmas are more generally taken into account. The break-through came with the discovery of the inverted $\mathrm{V}$ events (Fig.4).

There is now strong evidence that there are double layers with voltage drops $\Delta V$ of up to some kilovolts in the low magnetosphere (about $5000 \mathrm{~km}$ above the Earth), and that auroral electrons inpinging on the ionosphere are accelerated in these. We also know that the formation of such a double layer requires an electric current $I$, which (at least usually) should be flowing upwards in order to produce double layers. Often the power $P=I \Delta V$ released in the double layer is essentially delivered as high energy electrons $(C . P . I I .6)$.

* Detailed motivation for views expressed in this paper are given in the monograph Cosmic plasma. References are made to chapters and paragraphs $(1)$. 
It is known from laboratory that double layers may get unstable and "explode", which means that the voltage $\Delta V$ surges up to values many orders of magnitude larger than before explosion. Most of the energy $W=\frac{1}{2} I I^{2}$ of the circuit in which $I$ flows ( $L=$ inductance) may be transferred into high energy particles. Solar flares, magnetic substorms and the "folding umbrella" phenomenon in comets may be due to exploding double layers. A considerable part of the high energy particles observed in the magnetospheres may be produced by double layers (see C.P. II.6.4). During the last few years the interest in double layers in cosmical physics has avalanched. Still there are reasons to believe that we are only in the beginning of the exploration of their significance. For example, their role in more distant regions such as galactic and intergalactic space is not at all clarified.

As the Symposium on Plasma Double Layers at Ris $\phi$ will take place in a few days, we need not discuss the basic properties of double layers more in detail.

\section{B. Energy transfer}

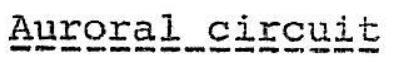

The mapping of the auroral circuit, started by Armstrong and zmuda in 1974, represents a major progress in cosmic plasma physics. Certainly from the time of Birkeland (which means the beginning of the century) it has been suggested that circuits of this type should exist, but the unquestionabie demonstration of their existence put this part of the theory of cosmic plasmas on a firm base. Fig.5 gives a very likely simplified model of the auroral circuit. The central body (the Earth) produces a dipole field $B$ and $B_{1}$ and $B_{2}$ are magnetic field lines from this body, $c_{1}, c_{2}$ is a plasma cloud near the equatorial plane moving with velocity $v$ in the sunward direction (out of the figure) producing an electromotive force

$$
V=\int_{C_{2}}^{C_{1}+} v+\vec{b} \cdot \vec{d}
$$

which gives rise to a current in the circuit $c_{1} a_{1} a_{2} c_{2} c_{1}$. 
In a double layer $D$ with the voltage drop $\Delta V_{D^{\prime}}$, the current releases energy at the rate $F^{\prime}=I \Delta V_{D}$, which essentially is used for accelerating auroral electrons.

The energy is transferred from $C$ to $D$ not by high energy particles and not by waves. Also, it is not transferred by magnetic merging or field reconnection because the magnetic field is essentially static. It is transferred by the electric circuit.

\section{LoGal_PIasma_properties and the cjrcuit}

Consider a tube through which there flows a certain current $I_{0}$ (Fig. 6). The voltage $v_{0}$ between its electrodes depend on the size and the shape of the tube, the chemical composition, density and magnetisation of the gas, etc. If the current changes from Io to $I$, the voltage changes from $V_{0}$ to $V$. If the change is small, we can use the Iinear approximation

$$
\mathrm{V}-\mathrm{V}_{\mathrm{O}}=\mathrm{R}\left(\mathrm{I}-\mathrm{I}_{\mathrm{O}}\right)
$$

where $\mathrm{R}$ is a constant which is called the internal resistance of the tube. It is well known that plasma tubes often have a negative resistance $(R<0)$.

Suppose that the tube is connected to a battery $v_{b}$ through a resistor $R_{C}$ and an inductance $I_{C}$. Then the circuit obeys the equation

$$
v_{b}=V+R_{C} I+I_{c} \frac{d I}{d t}
$$

or from (1)

$$
I_{C} \frac{d I}{d t}=\left(R+R_{C}\right)\left(I-I_{0}\right)+C
$$

where $C=V_{b}-V_{0}+R_{C} I_{0}$ is a constant.

If $R+R_{C}=0$ the plasma will be in equilibrium $V=V_{b}-I R_{C}$.

If $R+R_{C}>0$ the current will change unill a stable equilibrium

is reached. The time constant of the change depends on $\mathrm{I}_{\mathrm{C}}$.

If $R+R_{C}<0$ no equilibrium is possible. Depending on higher order terms in (1) the plasma may produce regular oscillations with a frequency which depends on $\mathrm{I}_{\mathrm{C}}$. 
The discharge may aiso be disxupted. In this case most of the inductive energy $\frac{1}{2} \mathrm{II}^{2}$ of the circuit may be delivered at the point of disruption in the plasma.

It is welliknown that all electric circuits with $I \neq 0$ are explosive. If distupted they deliver the inductive energy at. the point of disruption where it may produce a violent explosion. These examples show that the properties of a current-carry. ing plasma depends on the circuit of which it is a part. "Nota bene: If in a magnetized plasme, curl $B \neq 0$ it carries a current!) Hence, even if we know all the local properties of a plasma, e.g. its density, temperature, magnetisation, chemical composition, we cannot predict its behaviour.

The conclusion is that the properties of a curcent-carrying plasma cannot be described by local theories, such as magnetic mexging theories. It is necessary to consider the whole circuit in which the current flows in order to account for the properties of a plasma.

The explosive properties of all electric circuits are important in cosmical plasma physics. If the current through a plasina gets disrupted $e . g$. by the instability of a double layer, an explosion may occur. If the inductive energy in the circuit is large, as it often is in cosmic circuits, a very rapid and very violent explosion may occur. Examples are solar flares and magnetic substorms.

\section{Boundary conditions}

Consider a cosmic cloud (Eig.6). Iimited by a surface S. The properties of the plasma inside $s$ depend on the boundary conditions. Thus by changing the current through $s$, we can change the behaviour of the plasma. As was seen in the preceding paragraph, the properties of a plasma depend on the whole circuit in which the current flows. This means that we can describe the plasma inside $S$ by parameters inside $S$ on $S y$ if $I_{A}=0$ everywhere on $S$. Hence, even if we know all plasma parameters (like density, temperature, and magnetization) at every point inside $s$, we can describe the plasma properties theoretically only if there is no electric current crossing the surface. Therefore, the boundary problems have to be analysed very carefully. 


\section{"Magnetic merging" theories}

What we have fourd mears that we can describe plasma phenomena inside a finite volune only if no electric current crosses the surface. In the terminology of the magretic field description, this means that we can describe plasma phenomena inside a finite volume only if the perpend cular component of the curl it is zero at every point of the surface.

All theories of "magnetic merging" (ox "field line reconnection") which do not satisfy this cxiterion are misleading or erroneous, and deserve no attention.

C Dualism in cosmic plasma physics

Magnetic field versus electric current (particlel description Because it is easiex to measure magnetic fielos than electrio currents it is natural to present the results of space exploration (from spacecraft and from astrophysical observations) with pictures of the magnetic field configuration. Furthermore, in magnetohydrodynamic theories, it is convenient to eliminate the current and to represent the electric current density by curi $B$. This method is acceptable in the treatment of a number of pheromena which are related to plasma anisotropy and waves in plasmas (see Fig.7 and C.P. Fig. I.4).

However, there are aiso a number of phenomena which cannot be treated in this way, but which require an approach in which the electric current is taken account of explicitly. The translation between the magnetic field description and the electric current description is made with the help of Maxweli's first equation

$\nabla \times B=\mu_{0} i$

As shown in Fig. 6 it is essential to draw the circuits in which the currents flow which is equivalent to a description of: how the particles move.

The dualism between magnetic field and electric current description is somewhatsimilar to the general duaism in physics. To try to describe the transfer of ezergy in cosmic plasmas by magnetic merging is as hopeless as to describe the photoelectric effect by Maxwell's equation. 
D Application to solar wind - magretosphertc interaction.

\section{Simple model}

As an important example of the partiole (electric current) treatment let us consider a simple case illustrating transfer of energy from the solar wind to the earth's magnetosphere. We confine the discussion to the equatorial plane. We assume that far from the earth there is a constant southward directed magnetic field $\mathrm{B}_{z}=\mathrm{B}_{\mathrm{O}}$ and a constant electric field $E_{y}=E_{O}$. The solar wind moves with the constant velocity $v_{x}=v_{0}=E_{0} / B_{0}$.

In order to obtain a very simple nodel we substitute the magnetopause with an ideal conductor, so that the equipotential Iines in the solar wind are changed as shown in Fig. 8. Further we suppose that there is a current in the rnagnetopause which changes the northward directed terrestrial nagnetic field into a southward directed, which at large djstancos decreases to the solar wind value $B_{0}$. The motion of the solar wind is treated by the guiding centre method (of. for examle C.P. III.1.2). Hence the drift in the equatorial plane ts

$\overrightarrow{\mathrm{u}}=\left(\overrightarrow{\mathrm{F}}_{\mathrm{E}}+\overrightarrow{\mathrm{F}}_{\mathrm{i}}+\overrightarrow{\mathrm{H}}_{\mathrm{i}}\right) \mathrm{x} \overrightarrow{\mathrm{B}} / \mathrm{eB} \mathrm{B}^{2}$

with

$\overrightarrow{\mathrm{E}}_{E}=\mathrm{eE}^{\rightarrow}$ causing an electric drift

$\overrightarrow{\mathrm{F}}_{\mu}=-\mu \nabla \mathrm{B}$ causing a diamagnetic drift

$\vec{F}_{i}=-m \frac{d u}{d t}$ causing an inertia drift

In order to make the model as simple as possible we put the solar wind temperature $=0$ so that the diamagnetic drift is negligible. The electric drift is

$\mathrm{u}_{\mathrm{E}}=\mathrm{E} / \mathrm{B}$.

The inertia drift is

$u_{i}^{p}=-\frac{m}{e / B} \frac{d u}{d t}$ for protons and

$u_{i}^{e}=+\frac{m}{|e| B} \frac{d v}{d t}$ for electrons.

Although usualiy small the inertia drift is important because it produces a current $i_{i}=\ln \ln \left(u_{i}^{p}-u_{i}^{e}\right)=\frac{p}{E} \frac{d E}{\partial t}$

where $n$ is the number density of electrons and protons and $p=$ 
$=n\left(m_{p}+m_{e}\right)$ is the mass density. The electric drift is decelerated because when the plasma approaches the magnetopalise $B$ increases and $E$ decreases and hence the inertia current flows antiparallel to $\mathbb{E}$. This means that we have an electric dynamo. The current it produces is likely to close in the circuit shown in Fig. 8 (cf. C.P. III.6).

It is easily shown that the power $P=V$. I which this dynamo delivers to the circuit equals the loss in kinetic energy $\frac{\rho}{2} \frac{E^{2}}{\bar{B}}$ of the plasma due to its slowing down when it approaches the magnetopause.

If the magnetic field increases by a factor 3 in the inertia current region $(=$ shock front region) and the electric fileld decreases by a factor 2 , the plasma velocity decreases by a factor of 6 . Hence the kinetic energy of the plasma goes down by a factor 36 , so that $97 \%$ of i.ts kinetic energy is transferred to the circuit.

The inertia current dyramo produced at the deceleration of a plasma reprewents, the main mechanism for transfer of plasma kinetic energy into electric circuit energy. Magnetic merging is irrelevant because the process can take place even if the magnetic field is constant (C.P. II.3).

Fig. 8 shows how the inertia current is likely to close. Part of its power is used to accelerate the solar wind sideways (and backwards). As the plasma is collision-free, increased pressure in front of the magnetopause cannot give rise to a sideway deflection.

Approachhing_a_more_rea

From a simple model we shall now approach the real conditions in two respects:

If the solar wind temperature is so large that $F_{\mu}$ is not negligible a considerable part (often the major part) of the solar wind kinetjc energy is converted directly into heat. Further, in order to let the circuit transfer energy to the magnetopause we must give this a finite resistivity. This changes the equipotential lines somewhat, so that part of the plasma reaches the magnetosphere.

We must also take account of the energy tapped from the solar wind to the north and to the south of the equatorial plane. Part 
of it is transferred to the magnetosphere (see C.P. III.6). Hence the magnetosphere current does only in part derive from the circuit of Fig.8.

our results can be generalized. It seems that the main mechanism of energy exchange - in both directjons! - between a moving plasma and a circuit can be treated as a result of inertia currents. As pointed out above magnetic merging theories are misleading or erroneous (except in special cases).

E Newtonian attraction and electromagnetic attraction

A mass element $d m_{1}$ is attracted by another mass element $d m_{2}$ at distance $R$ (counted from $d m_{1}$ to $d m_{2}$ ) by a Newtonian force $\mathrm{d}^{2}{ }_{\mathrm{N}}=\mathrm{G} d \mathrm{~m}_{1} \quad \mathrm{~d} \mathrm{~m}_{2} \frac{\overrightarrow{\mathrm{R}}}{\mathrm{R}^{j}}$

( $G$ = gravitational constant). Besides that there is also an electromagnetic force

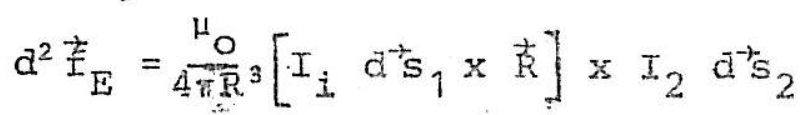

caused by the current $I_{2} d s_{2}$ acting on the current element $I_{1} d \vec{s}_{1}$ and $\vec{r}$ is the vector from $\vec{d}_{s_{2}}$ to $d \vec{s}_{1}$. For infinite parallel currents the force $E_{\mathrm{E}} / \&$ per length $\&$ of the conductors is

$$
f_{E} / l=\frac{2 \mu_{0} I_{1} I_{2}}{4 \pi R}
$$

Compared to the Newtonian graviEation the electromagnetic attraction is negligible for condensed matter (like stars, planets and satelitites). In dispersed media it is often dominating. Contrary to the Newtonian gravitation which tends to produce spherical structures the electromagnetic attraction tends to produce filaments, or in some cases, thin surfaces carrying currents.

\section{Filamentary structure of cosmic plasmas}

Since long it j.s wellknown that filamentary structures are very often observed (see C.P. II.4). Examples are

coronal streamers and prominences in the solar atmosphere, and filaments in galactic clouds.

There are rather good (although not fully convincinglarguments that all (or at least most) filamentary structures are produced by electric currents. 
Examples of filamentary structures are given in Fig. 9,10,11, and 12. Of these 11 and 12 are taken from E.E. Barnard.s atlas (2). He tried to atcract attention to the "thin dark "lanes" of uniform width". He admits that "it is probabie that some are due to pure chance" but gives convincing arguments for the view that "others are real and are due to some law that wili reveal fisejf in the course of timen .

During the more than 50 years after the publication, these conspicuous results have attracted very little attention. The reason seems to be that the orthodox education of astrophysicists includes Newtonian mechanichs but not plasma physics. Hence the astronomers did not note that in 1934 Bennet published his paper on "Magnetically self-focussing streans". It is quite possible that his theory will lead to the law Barnard was looking for. Instead these conspicuous "lanes" for with our teminology "filaments") were very seldor mentioned. Indeed they were swept under the rug and have remianed there for half a century.

The neglected pinch effect

The simple formula, for calculation the gas pressure gradient $p$ in a stationary plasma is

$\nabla\left(P+E^{2} / 2 \mu_{0}\right)-\frac{(B \nabla) B}{\mu_{0}}=0$

Under certain assumptions (for example, if all magnetic field lines are paraliel) the right hand term is zero and we have $p+B^{2} / 2 \mu_{0}=$ const.

The general belief that a magnetic field counteracts the contraction of a cloud is essentially based on this formuia, which is valid in only one special case.

For reasons which are difficult to understand - and still. more difficult to defend - the third term is almost always neglected in the astrophysical Iiterature. The result is that the pinch effect - or more generally-.. the electromagnetic attraction is systematically neglected.

This means that for example the generally accepted theories of the evolutionary history of intexstellar clouds, the formation of stars and solar systeme are based on a misunderstanding. 
An example of the importance of the pinch effect term is given in Fig. 13 (see Cosmic Elasma IV.8).

F Cellular structure of seace

Current layers in the magretospheres

To most plasma physicists (except Dungey who at least in part had predicted it) the discovery of the magnetopause was a shock. It demonstrated that far up in the magnetosphere there is a thin current layer which at least in some respects has a high degree of persistence (in spite of the fact that it seems to be in continual motion). It separates regions with different magnetisation, different pressure, temperature and chemical composition. Hence we can regard it as a cell wall separating regions of space with widely different properties. (See C.P. II.9.5).

A similar layer is also found in the magnetotail.

Similar current surfaces are found around other planets, most pronounced around Jupiter and saturn. There are probably at least one such surface in comets. Moreover, in the solar wind there is a surface current, esscutially in the equatorial plane but waving up and down "ballerina effect"). This current layer separates a northern region with inward magnetic field from a southern region with outward directed magnetic field for vice versa depending on the solar cycle).

Current layers are sometimes very thin. For example the thickness of the magnetopause may be as thin as a fraction of an ion Larmor: radius. It moves often as rapidly as $25 \mathrm{~km} / \mathrm{sec}$, and is usually wavy. (3).

Cell_walls in the magnetospheres and in more distant_regions It is almost impossible to observe a cell wall before penetrating it. When a spacecraft approaches a wall, the instruments cannot tell its existence until suddenly the readings of a number of its meters change.

Current layers acting as cell walls are found in all those region: accessible to space crafts, but because of the mentioned proper- 
ties of the walls we have obviously no possibility to observe them outside the reach of spacecrate.

Do similar walls exist far out in the galaxy? rhere are no such phenomena observed, nor should we expect to observe them if they exist because we know that they are not observable unless a measuring device penetrates them.

It is unpleasant to postulate the existence of cellular structures in the galary if we have no possibilities to observe them. However, it is obviously still more unpieasant to postulate that a basic property of space changes abruptiy at the present outer reach of spacecraft.

The conclusion must be that space is very ilkely to possess a general cellular structure. Hence we must conclude that also out in galactic and extra-galactic space there are walls separating regions of different nagnetisation density, temperature, chemical composition and maybe different kind of mateer (koinomatter - antimatter). (See C.P. TI. YO. IV.9.6, VI.3.A.)

Space in space age

Fifty years ago space between planets and stars was considered as the most perfect vacuun. This was correct in the aense that its density is several orders of magnitude lower than the best laboratory vacuum. However because of the large dimensions even a very low density is impotant.

When interstellar matter was discovered, it was thought to be structureless, homogeneous, nebulous.

This concept is now replaced by the space age concept of space. As we have seen space can be divided by a number of "cell-. "Walls" consisting of electric cirrent layers. Further, space is penetrated by current produced filaments which often have densities and magnetisations several orders above that of interfilamentary space. Their tempexature may be either higher or lower than in interfilamentary space.

What is said is applicable both to the low density interstellar space and to interstejlar clouds. 
Tsytovich (4) has draw attention to some interesting properties of filamentary curients in space. Whey may produce exploding double layers in random regions, cesulting in very large tran sient releases of energy and production of strong local electric fields. Whis nay git 3 wise to a stc hastic acceleration of cosmic rays. Maybe the microvave background radiation - which now is the only observational support of the big bang may be generated by processes or this kind.

III Application of the new paradigm to cosmology and solar system evolution

A The new concept of space and the existence of antimattex There is no doubt that the red shift of adaxies is due to a "cosmological" Hubbie expansion. Whether the red shifts of quasars, sometimes oxtremely large, also are "cosnological" is a controversial guestion (see C.P.VI.4.1).

The question whether there exist lavge quantities of antimatter in the universe has cone into another light by the djscovery of the celluiar structure of space. It 2 s quite possibie that some of the cell walls separate regions of antimetter from regions of koinomatter (orlinary natter). This means that the Leidenfrost layers separating the two kinds of matter are at the same time current layers. This makes us more confident that the layers have a high decroe of gtability (cF. Eig, 15).

It has been show that wh space age concept of space there are no valid arquments aganet he existerce of antinatter. The universe may vex. well be symetric incieed even every gelaxy may be symetrio with regard to koino- and antimatex. Ssee C. B. IV.9 and VI.2.2.)

B Annihilation as a source of energy

Energy release in cuesare

The quasars release. st moh enexuy that nuclear energy is by fax insufficient as ererg, worce. he have three possible ways to account for theil supply of energy: 


\section{Gravitation}

2. Annihilation

3. New laws of physics

As our analysis has started from the assumption that the basic properties of cosmic plasma are t're same everywhere, we camot accept (3)

In the choice between (1) and (2) It should be observed that quasars normally eject nattex, often as jets. The rejease of gravitational energy is basically connected with a fali-in of matter, e.g. towards a "black hole" (if such objects exist) whereas anihilation should result in ejection of secondary products. Hence (2) should be erefersed.

More arguments for this view have been given elsewhere (C. P. GI):. .. It is concluded that every galaxy is Iikely to contain both kinds of matter. (C.P.VI.2.)

The Hubbje expansion

The kinetic energy of the Hubjle expansion constitutes the largest energy in the universe except the rest mass. Again, the source of this energy may be (1), (2) or (3) according to above. As there is no doubt hat the expansion results in a lower gravitational potential (I) is excluded. Fence we have the choice between (2) and (3). (see C.P.VI.5.2.)

The big bang cosmology accepts (3). It is postulated that at time as 0 processes occ rred wich are completely different from the processes we have studied in other connections. Some believers ir big bang adnit openly that it is necessary to accept a divine intervention but most prefer not to discuss this sensitive guestion explicitiy.

As our basic assumption is irreconcilable with such an hypothesis, the only alternative which remains for us is (2). This means that the finetic energy of the fubbje expansion ultimately derives from annihilatior. The more detalled analysis indicates that the release of annihilation energy possibiy occurred essentialiy when the size of the metagalaxy was about $10^{25} \mathrm{~m}\left\{=10^{9}\right.$ icht years which neans about 10 of of present size. (CP VI.5.) 


\section{Approach to cosmology}

Should the bjg bang hypothesis_be considered as sacrosanct?

When the big bang hypothesis was new it was claimed that there were a number of observational facts supporting it. Further studies have eroded the observational support and the only phenomonon on which big bang is based now is the cosmic microwave background radiation. ISome people claim also that big bang is necessary in order to explain the high helium abundance, but iff real, this may probably be explained also in other ways.) Concerning the microwave radiation this would be a very strong argument if space were empty and structureless. The strength of the argument in a highiy structured space which we have found. above, is less convincing. In fact, of the whole electromagnetic spectrum from long radio waves to short $\gamma$-rays the millimeter region is the region which is most poorly investigated. We do not know how much interstellar and intergalactic matter absorb milineter radiation, because we know very little about its chemical and structural. composition. Hence we cannot exclude that the microwave background radiation is generated or made isotropic in space.

Confrontation between three-dinensional highy y structured model and fourmamensione homogeneous model

observitions have demonstr ited that the mittagalaxy has a hierarchical structure out to at least $10^{25}$ m.(C.P.VI.1.3.2.) This means essentially that It should be possible to extrapolate the laws we have derived from laboratory and magnetospheric measurements out to at least $10^{25} \mathrm{~m}$. Doing so we remain the whole time very far from the Iaplace-Schwarzschild limit, which means that we do not need to take general relativity into account. Space remains basically Euclidean and highly inhomogeneous (5).

If we accept the big bang hypothesis we must assume that somewhere between $10^{25} \mathrm{~m}$ and $10^{26} \mathrm{~m}$ the character of space should change from three-dimensional and highly structurized to four-dimensional and homogeneous. As this region is not yet explored we are unable to make a decision now. 
If we do not accept the big bang hypothesis we can account for the metagalaxy as a new level in the hierarchy which then should include galaxies, galactic clusters, superclusters and finally the metagalaxy. urther, as pointed out in III B decades of ambitions attempts to explain the enourmous energy release in quasars as due to other sources then annihilation have not been very successful. Hence in principle the observed properties of quasars are a good argument a symmetric cosmology. If we accept this we can also account for the energy of the Hubble expansion without introducing new laws of physics.

D Evolution of interstellar clouds into stars, planets and satellites

The traditional ("Laplacean") approach to the evolution of interstellar clouds and the formation of stars, planets and satellites neglects four plasma effects which according to the new paradigm must be introduced: The theories of dusty plasmas, of the pinch effect, of the critical velocity and the plasma-planetesimal transition with a $2: 3$ contraction.

Dusty plasma

As interstellar clouds usually contain considerable amounts of dust, the theory of dusty plasmas is essential. Unfortunately there are not yet any in situ measurement in a dusty plasma in space. Such investigations will probably take place when spice probes are sent through cometary tajls which are the only region in our neighbourhood which consist of dusty plasma. However laboratory measurements of the interaction between plasmas and dust (or at least small probes) give us some confidence that we understand at least some of their properties (6)

It is possible that the combined action of gravitation and electromagnetic effects in dusty clouds will lead to the formation of growing dust balls. When they have become large enough (Ferhaps about lunar size) they will serve as nuclei for a run-away accretion. Later a large number of such "stellesimals" may form stars through aggregation similar to the pianetesimal accretion (C.P:V.8). 


\section{Pinch effect}

It is weli-lnown that at least many clouds posses a filamentary structure, which may indicate significant electric currents in them. It is possible that the clouds are formed by a combined action of electromagnetic and gravitational forces. In other words the finch effect of currents through space, which we have discussed above, may concentrate the dilute interstellar matter into clouds, but of course gravitation will also be importan In any case the usually accepted theory that stars are formed through a Jeans' collapse is not necessarily correct. Introduction of the theory of dusty plasmas and of electromagnetic contraction changes the background drastically.

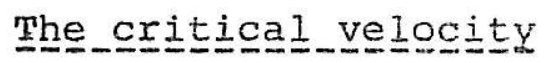

If the planets and satelities in the solar system are plotted as a function of the gravitational potential in relation to their central bodies, it is found that different groups of bodies are located at certain discrete values ("band structure" of the solar system)(C.P.V.7). This can be explained if matter falling in from infinity is stopped when its velocity of fall has reached certain critical values. When this was discussed through an araIysis of the structure of the solar system no physical effect was known which could produce such a result. This inspired laboratory experiments in order to find whether such an effect existed or not. The experiments werf successful: tre required effect, called "the critical velocity", was found. The effect means that when the relative velocity between a neutral gas and a magnetized plasma reaches certain critical values, the interaction between the media suddenly increases by orders of magnitude. (C.P.IV.6.) Many experiments at different laboratories have confirmed the existence of the "critical velocity" phenomenon. The theory of it has made significant progress but is not yet completely satisfactory.

This is one of the rare cases when an observed cosmogonic fact has lead to the discovery of a previously unknown laboratory effect.

Transition from_plasma to planetestma]s_and the 2 s 3 contraction A plasma, dusty or not, which is located in the magnetic dipole field of a rotating gravitating body, will be brought into partial 
corotation by electromagnetic transfer of momentum. Its velocity is then determined by the equilibrium between gravitation, centrifugal force and electro-magnetic forces. At neutralisation the latter vanish and the result is that the mediun contracts by a factor $2: 3$.

The effect of such a transition from a piasma state to a planetesimal state is clearly found in the Saturnian ring systen, where the bulk structure is determined by the $2: 3$ contraction ("cosmogonic shadow effect"). It is also found in the asteroid belt (7).

The band structure and the cosmogonic shadow effect are of essential importance for the understanding of the evolutionary history of the solar system. But, of course, a large amount of work is necessary before a clear picture can be obtained.

It seems evident that plasma effects are decisive for the evolution from an interstellar cloud up to the rather well defined transition from the plasma state to the planetesimal state which is marked by the $2: 3$ contraction. From there plasma effects are of no impoxtance. The planetesimals delivered as a result of this transition accrete to planets exclusively by mecharical processes.

\section{Final remarks}

When NASA started its systematic space investigations it stated that such a large enterprise could be justified only as an attempt to clarify basic scientific and "philosophical" questions such as three cardinal problems: the origin of the solar system, the origin (if any) of the universe, and the origin of life. After the negative results of the Viking project the hope of detecting extraterrestrial life is close to zero, and NASA:s future contribution to the solution of the problem of the origin of life will be essentially indirect.

Concerning the first two cardinal problems an enourmous amount of data has been collected which may be of importance. However, in order to derive scientific knowledge from this mass of data, it is necessary to know the relevant laws of physics. As we have shown both cardinal problems belong at least in part to plasma physics. 
This means that their solution depends on basic laws of plasma physics which essentialiy are clarified by in situ measurements in the magnetosphere. Hence magnetospheric research has been and is a conditio sine qua non for clarifying the origin of the solar system and the origin (if any) of the universe.

But also in other respects magnetospheric investigations have been and are essential for these cardinal problems: it has educated a considerable number of scientists in new methods of approach which are essential in order to solve plasma problems. This means that the plasma physicists may be the only group of scientists from whom we can expect real progress in research on the origin of the solar system and on cosmology (but this contribution will not be of real value before the paradigm transition has been completed). This unique position gives us all a ceep responsibility for the progress in these fascinating fields of research.

Acknowledgements I wish to thank several collegues, including Drs C.-G. Fälthammar, N. Herlofson and F. Carlqvist for discussions, and Mrs E. Florman, Ko Vikbladh and Kai Forsberg for the editing. 


\section{References}

(1) A review of the investigations which are leading to the paradigm change is given in $H$. Alfvén, Cosmic Plasma, 1981, Dordrecht, Holland: D. Reidel Publ. Co. The page references refer to this monograph. Only refereices which are not found in C.P. are listed here.

(2) Barnard, E.E., A Photographic Atlas of Selected Regions of the Milky Way, Ed. E.B. Frost, Carnegie Institution of Washington 1927.

(3) Williams, D.J., Magnetopause Characteristics at 0840-1040 Hours Local Time, JGR $85,3387,1980$.

(4) Tsytovich, V.N., Private communication, 1982.

(5) Alfvên, H., On Hierarchical Cosmology, TRITA-EPP-82-03, Dept of Plasma Physics, Royal. Tnstitute of Technology, Stockholm, Sweden.

(6) Mendis, D.A., Houpis, H.I.F。 and Hill, J.R., The GravitoElectrodynamics of Charged Dust of Planetary Magnetospheres, JGR, 1982 (in press).

(7) Carlquist, P. and Alfvên, H., Transition from the Dusty Plasma State to the Planetesimal State of the Early Solar System, TRITA-EPP-81-09, Dept of Plasma Physics, Royal Institute of Technology, Stockholm, Sweden.

The Solar System and Its Exploration. Papers presented at the Alpbach Summer School, $29 \mathrm{July}-7$ August 1981, ESA SP-164:

(a) Alfven, H., 1976, The Saturnian Rings, Astrophys. Space Sci. 43, 97, 1976.

(b) Alfvén, H., 1981a, origin of the solar System. Introductory lettex at the Cospar Budapest Meeting, Adv. Space Res., 1, 5, 1981.

(c) Alfvén, H., 1981b, The Voyagex 1/Saturn Incounter and the Cosmogonic Shadow Effect, TRITA-EPP-81-01, Dept of Plasma Physics, Royal institute of Technology, Stockholm, Sweden,

(8) Torvén, S. and Iindberg, I., Properties of a Fluctuating Double Layer in a Magnetized Plasma Colum, J.Phys. D: Appl. Phys. 13, 2285, 1980 . 


\section{Figure Captions}

\section{Fig. 1 Iinear size of plasmas}

Laboratory and nagnetospheric plasmas can be investigated by sophisticated diagnostics. Cosmic plasma physics should be based on research in these regions. Theories of regions outside the reach of spacecrafts are necessarily more speculative. They should be based on extrapolations of results from the accessible regions. The sun and comets are also outside the accessable regions.

Fig. 2 Plasma experiment for investigating the properties of a double layer From a mercury plasma produced by a discharge, a plasma column confined by magnetic field penetrates to the anode. In this current-carrying column a double layer is produced as soon as the current exceeds a certain limit

Fig. 3 Voltage $V$ and density $n$ along a plasma column Below a certain current $I_{0}$ these are slowly varying functions of $z$. If the current is rajsed by a few percent, a double layer is produced, at which the voltage increases by 30 volt in a very small distance. The plasma density also changes abruptly. (8).

Fig. 4 "Inverted V" events (Gurnett, 1972)

Right: Variation in the electron energy measured by a spacecraft, indicating the existence of electric fields $E_{1}$. Left: Electrostatic potential distribution. The magnetic field is vertical. The current-carrying flux tube is "insulated" from the surrounding plasma by a thin cylindrical shell of rotating plasma, which produces a voltage drop which equals the electrostatic drop in the layer. Compare the equipotential lines with those in Fig. 2. Both are examples of "cable" formation in a plasma. 
Fig. 5 The auroral circutt seen fron the sur fstmplizied picture).

The central body (Earth and fonosphere) maintains a dipoletiela. $B_{1}$ and $B_{2}$ are magnetic field lines from the body. o is a plasma cloud near the equatorial plane moving in the sunward direction cont of the figurel producing an electromotive force $v=\int_{c_{2}}^{c_{1}} \bar{x}$. dis wheh gives rise to a current in the circut c1, a1, a2, c2 $c$. In a double layer $D$ with the voltage $V_{D}$, the current releases energy at the rate $P=I V_{D}$, which essentially is used for accelerating auroral electrons. The energy is transferred from o to $\mathrm{D}$ not by high energy particles or waves, and not by magretio merging or field reconnection. It is a property of the electric circuit.

Fig. 6 Plasma in a laboratory tube and in a cosmic cloud as elements in electric circuits.

Fig. 7 Dualism in Cosmic Plasma Physics

Fig. 8 Simplified particle model of solar wind impiaging on the magnetosphere. Equatorial plane.

Magnetic field southwards (Anto the plane). Electric eguipotential lines, along which the plasma drifts, are shown. When solar wind approaches the magnetopause, increase in $B$ and decrease in $\mathrm{E}$ slows it dow, hence productng inexti drift curents, antiparaliel to the electrid field. This means that there is an electric dynamo. The currents from it close through ragnetopause as show in the figure. Similar mechanisms to the north and to the south ot the equatorial plane close partaliy through the magnetopause, producing its nain current (ct. C.P. III.6. Fig. IIT. $14 \mathrm{a}$ and b). The model illustrates that thetrars.. fer of energy between plasma motion and electric cir. cuits occurs through inerita curcent aynamos. 
Fig. 9a Solar corona (March 1970). The Eilamentary structure is probabiy caused by electric currents.

b Detail of the Veil nebula.

Fig.10a,bFilamentary structures are much more comon than ususally thought as shown by comparing the ordinary photograph of the Lagoon nebula in Sagittarius (a) with the samo photograph subjected to contrast enhancement (b).

c Contrast-enhanced photograph of the orion nebula.

Fig.11 Filamentary structures in the dark nebula Barnard 72.

Fig.12 Filamentary structures at $a=16^{\mathrm{h}} 44^{\text {in }} \delta-22: 5$ (Earnard)

Fig. 13 Three special cases of stationary and oylindricaly surmetric current ( $i$ ) and magnetic fieId (B) configurations. (a) A toroidal current and an axial magnetic field leading to a force opposing contraction. (b) A force-free configuration with $i$ and $B$ pataliel. (c) The Eennett pinch with an axial current and a toroidal magnetic field.

Fig. 14 Northward component of magnetic field in the Earth"s equatorial plare.

Below: Magnetic field registration in a spacecratt which penetrates the magnetopause when moving outwards.

Fï. 15 A "Leidenfrost laver" separating a region of antimatier from a region of kojnomatter.

Annihilation at the interface between regions of boinomatter and antimatter causes a very hot intermediate surface layer which pushes the two kinds of matter apart. 


\section{COSMIC TRIPLE JUMP}

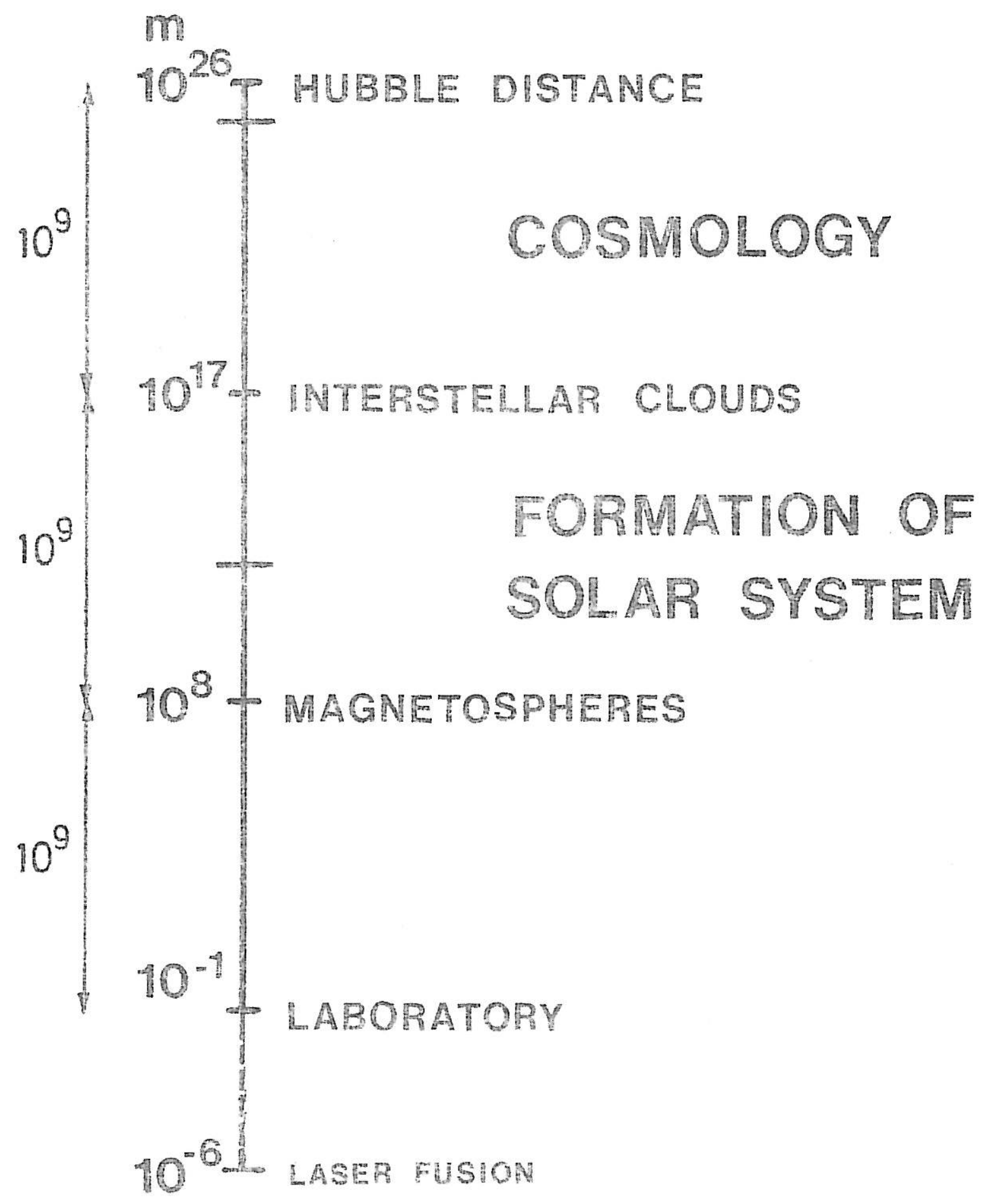



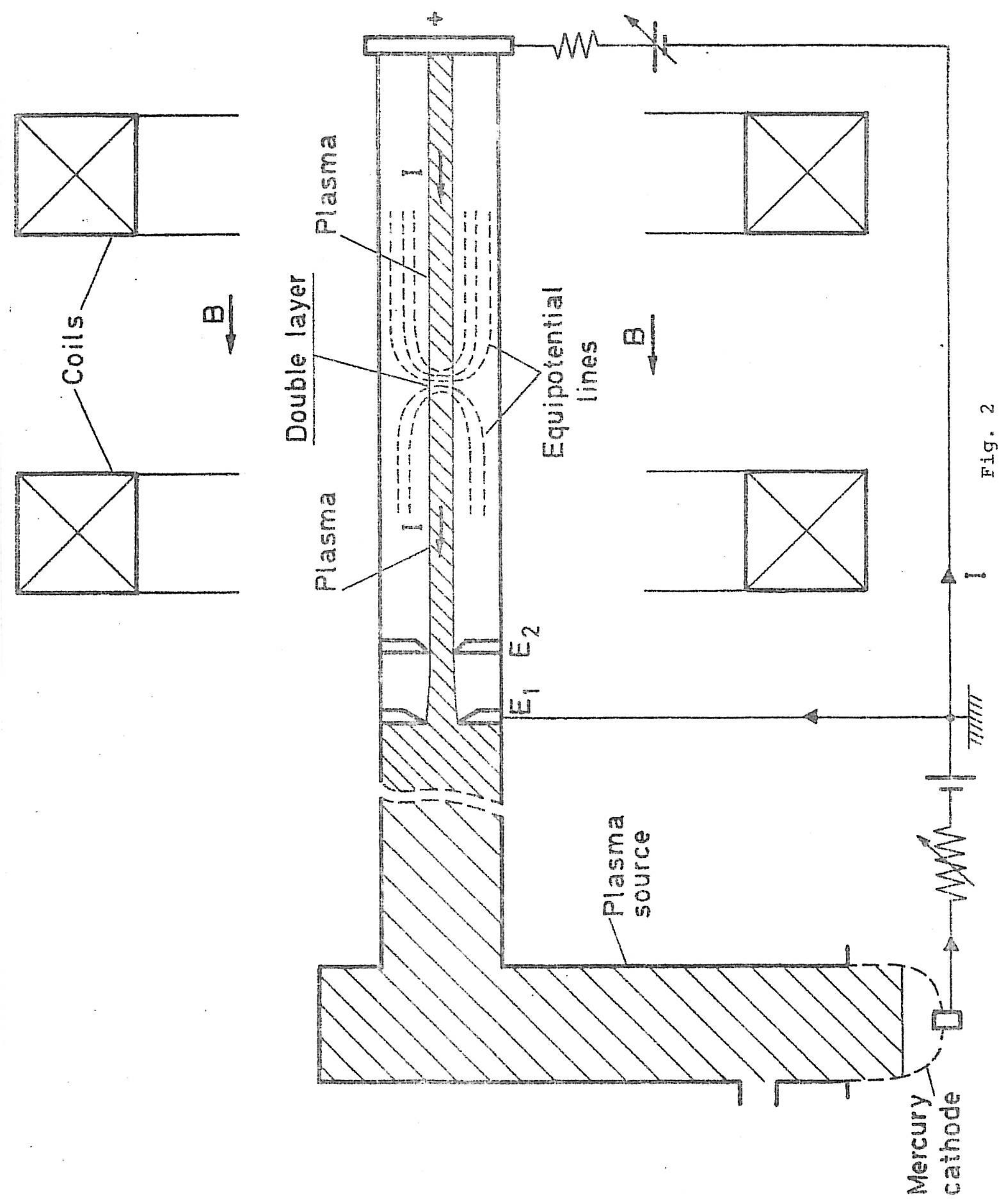

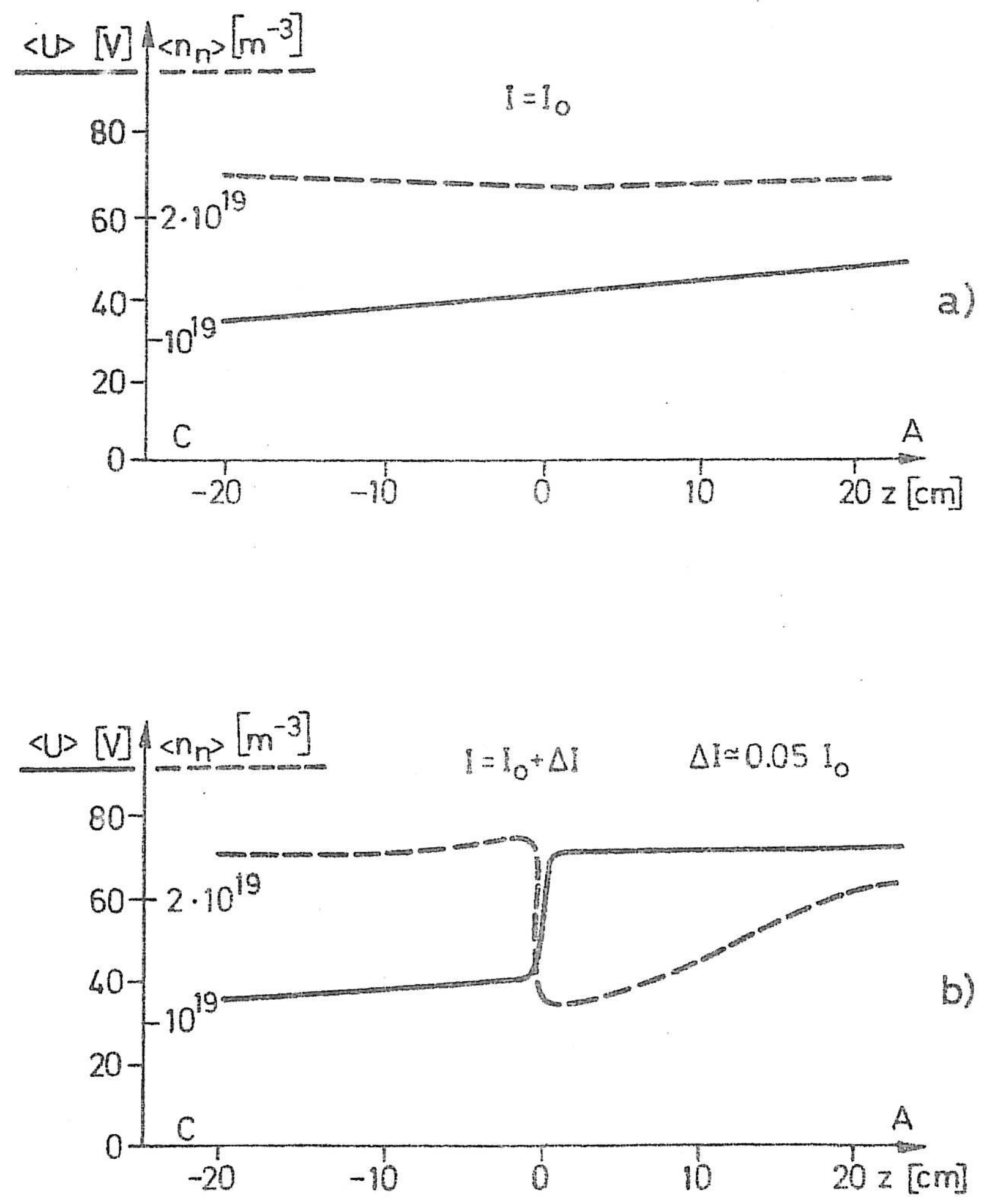

Fig. 3 


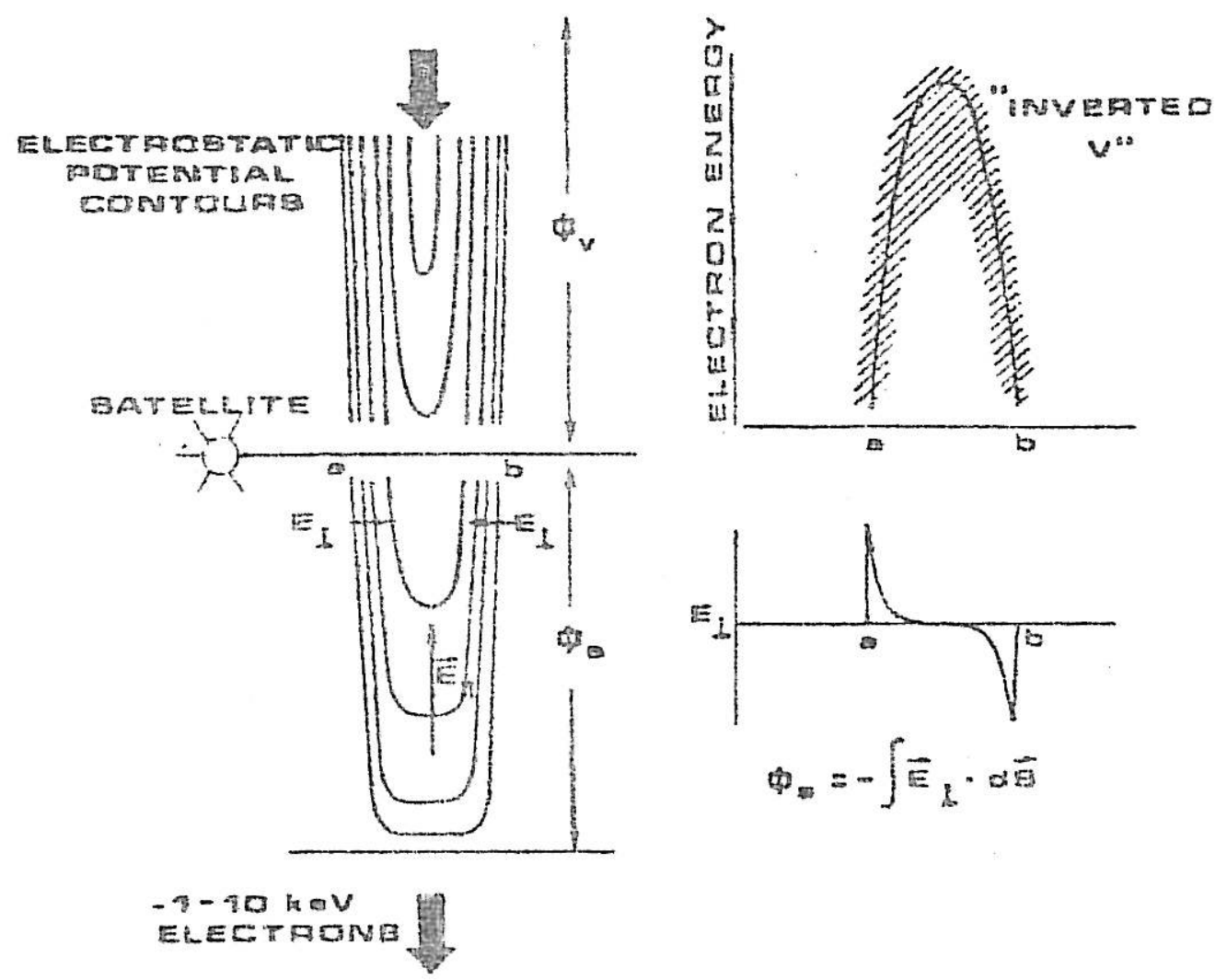

Fig. 4 


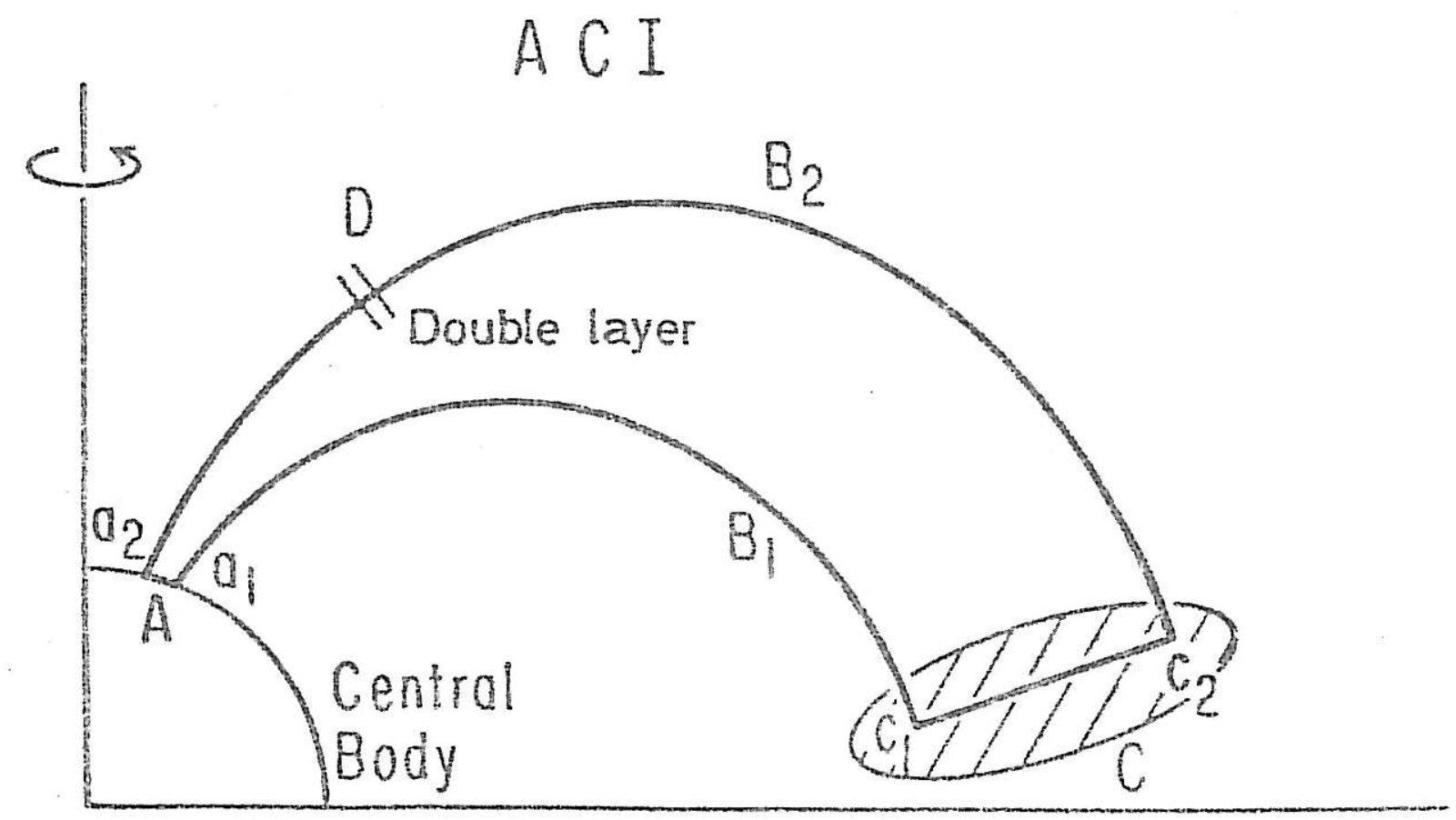

Fig. 5 


\section{COSMIC CIRCUIT}

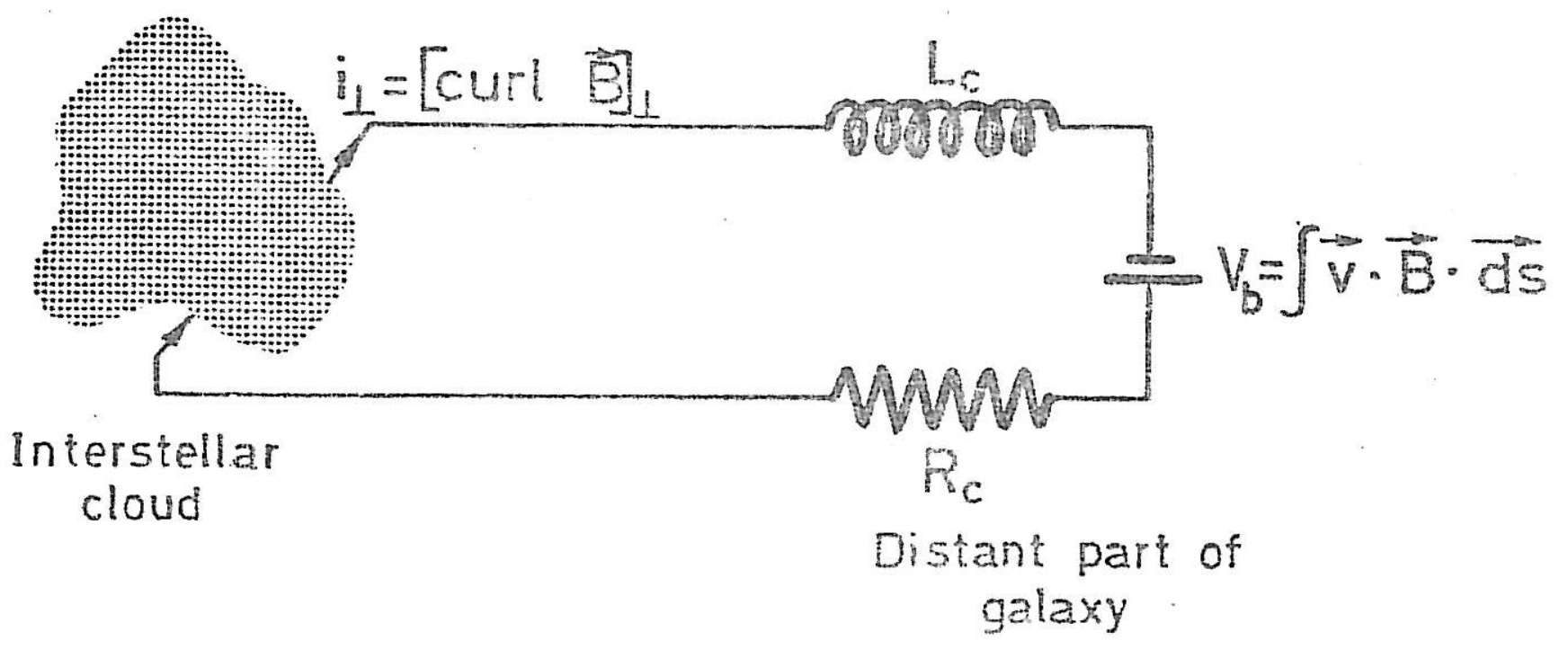

LABORATORY

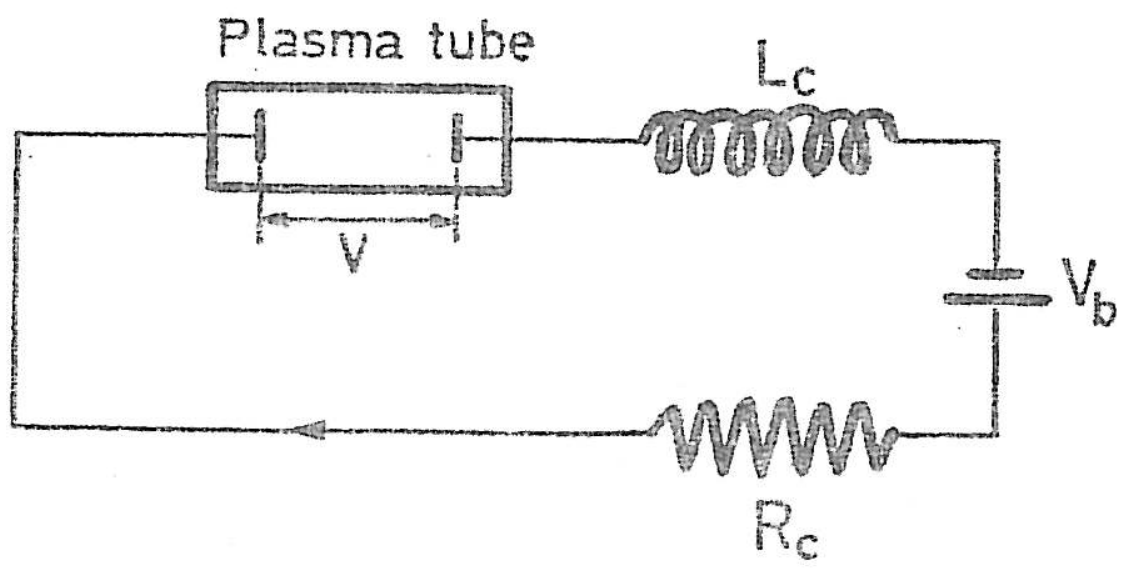

Fig. 5 


\title{
DUALISM IN COSUMC PLASMA PHYSICS
}

\author{
TRANSLATION FORMULA \\ $\nabla \times B=\mu_{0} i$
}

MAGNETIC FIELD DESCRIPTION

ESSENTIAL FOR

ANISOTROPY OF PLASMAS

WAVES IN PLASMAS
PARTICLE IELECTRIC CURRENT DESCRIPTION

ESSENTIAL FOR

FORMATION OF DOUBLE LAYERS

TRANSFER OF ENERGY FROM

ONE REGION TO ANOTHER

TO TREAT ENERGY TRANSFER BY MAGNETIC MERGING

(RECONNECTION) METHOOS IS BASICALL MRONG (EXCEP in some special cases).

IT IS AS IMPOSSIBLE AS DESCRIBNG THE PHOTOELECTRIC 


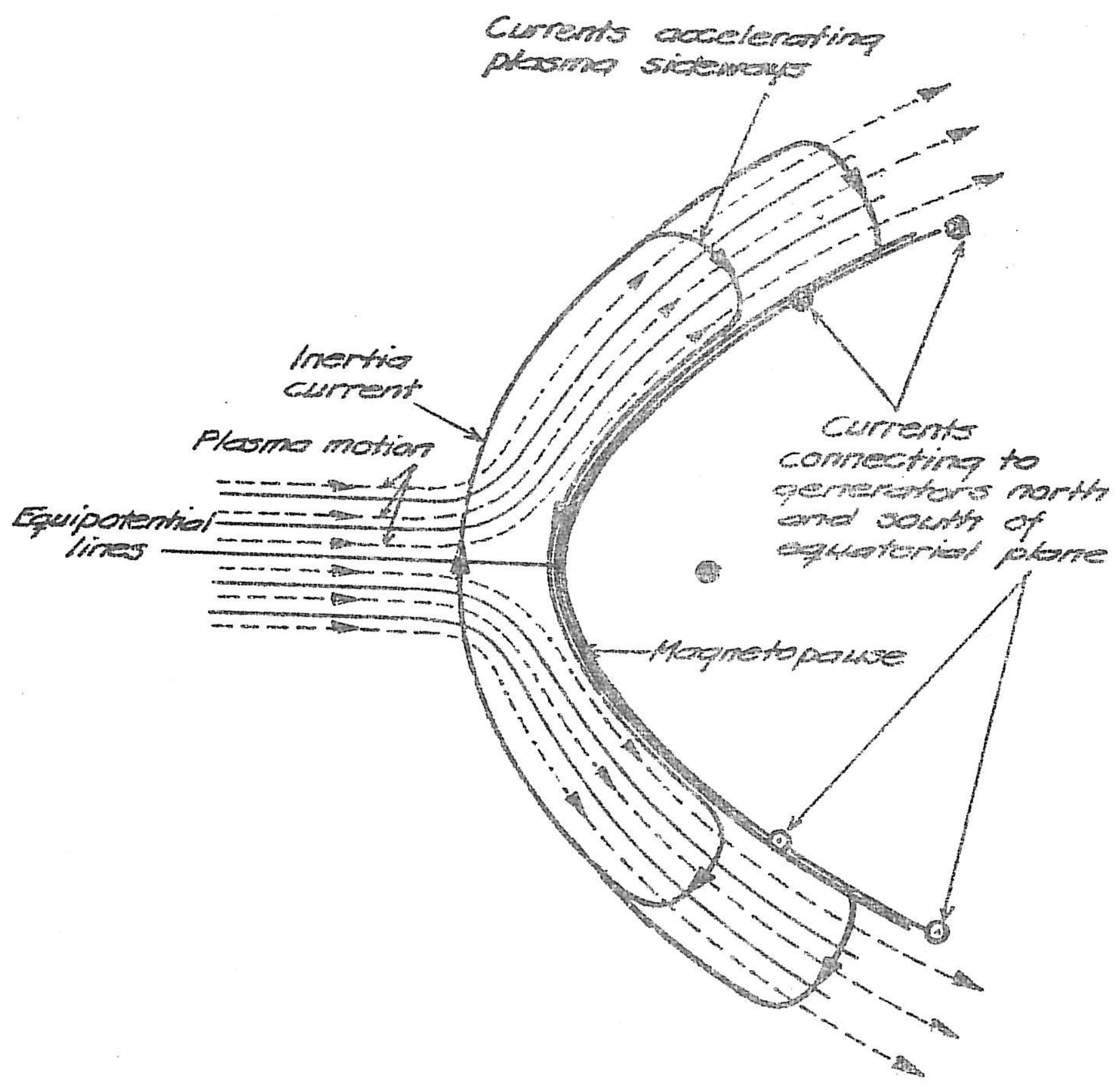

Fig. 8 


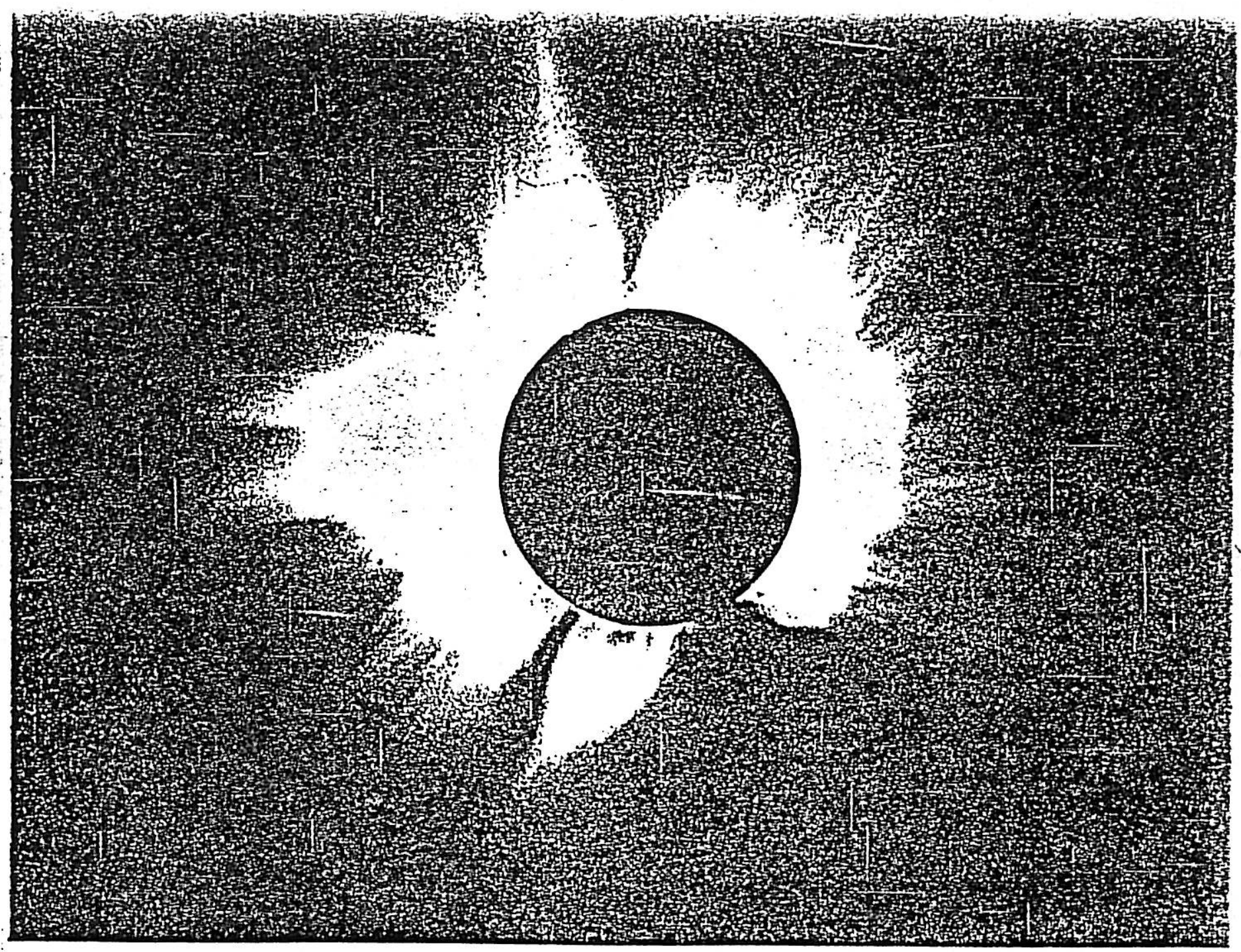

H.

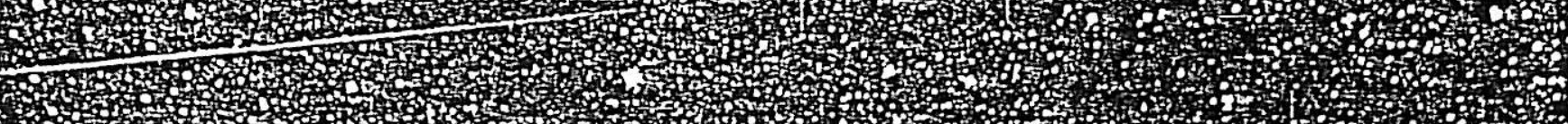
6 (3) S.7. H.7. 4 (2)

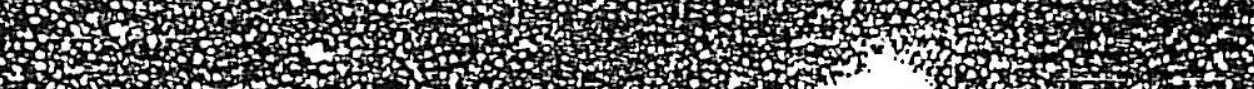

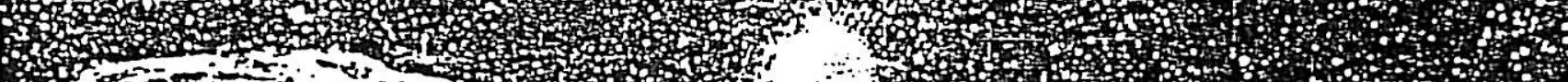
(2)

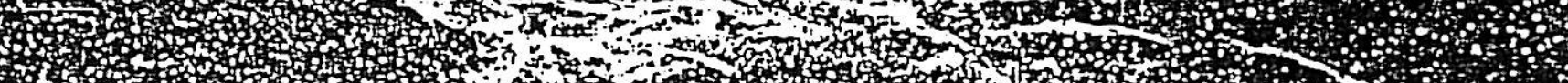

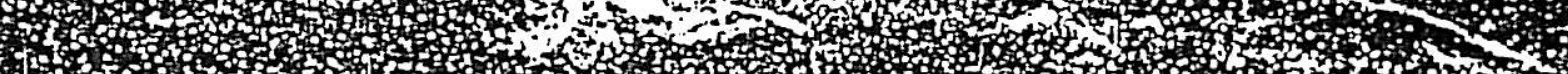

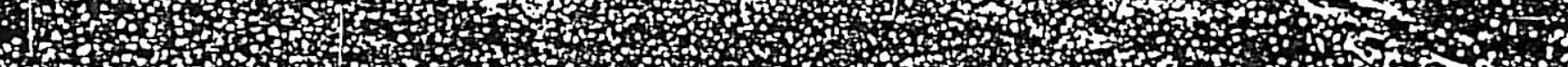
H.

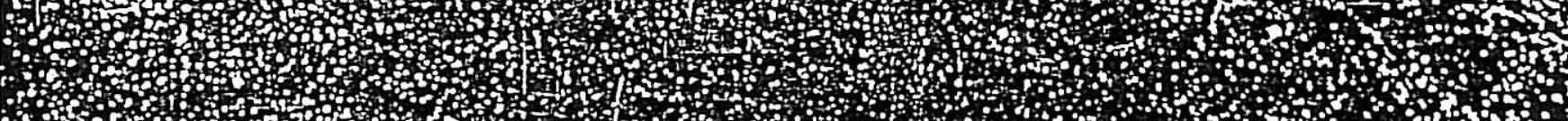
(3)

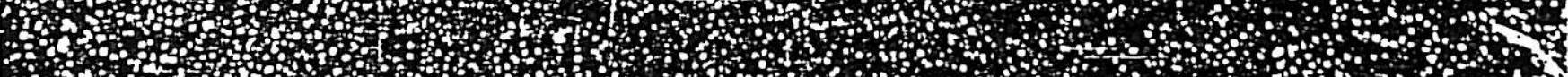

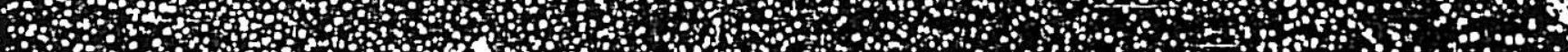
(1) (2)

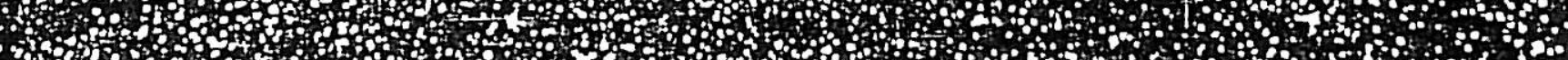

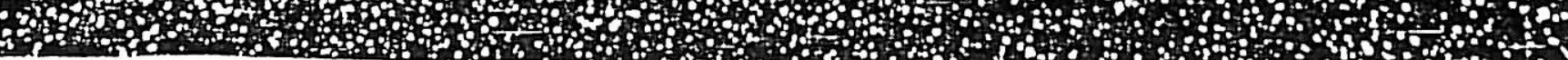




\section{3) \\ 40150}

ecting

sis

2

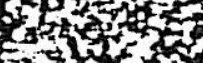

iristix

1. 10

ixt?

17030

(1)

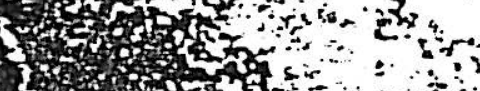

80250

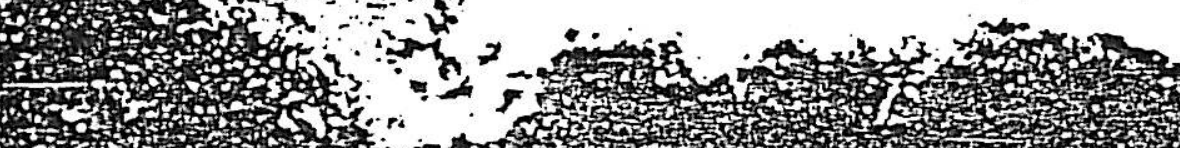
7.
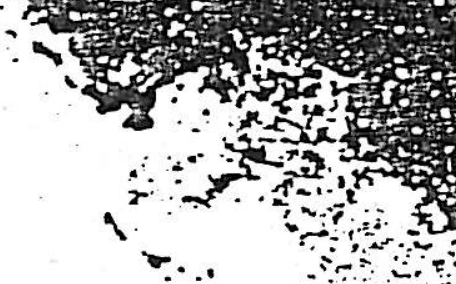

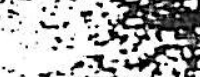

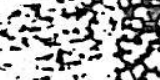

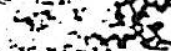

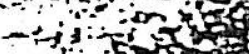

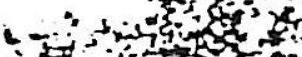

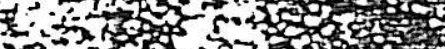

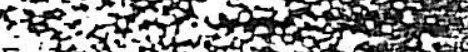

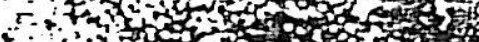

$x-2$.

- o

$\therefore=$ 1

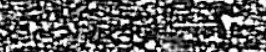

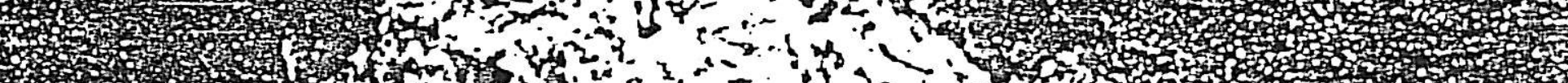

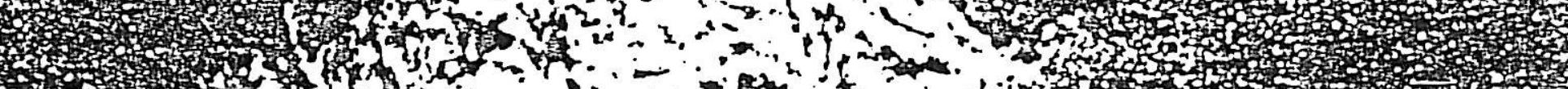

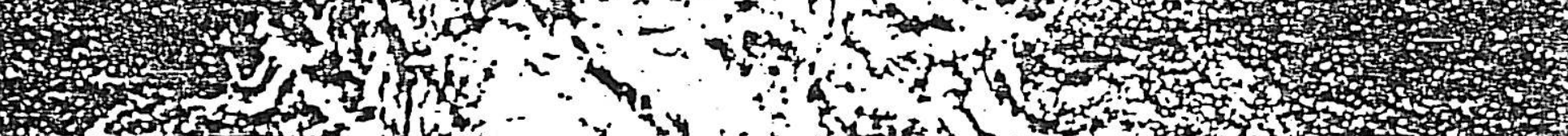
(2) 54 (3) 50.

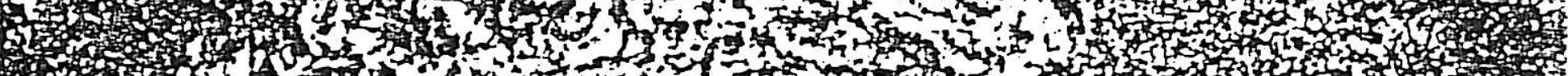

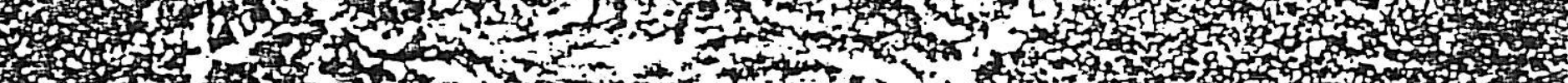

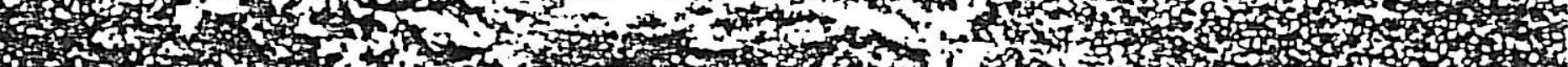

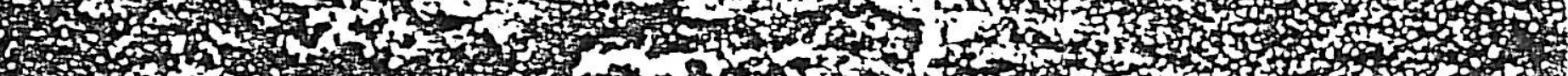

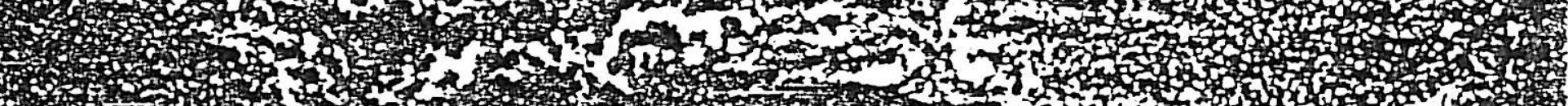

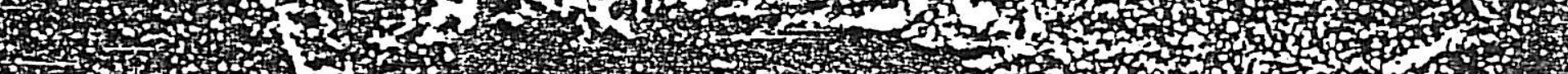

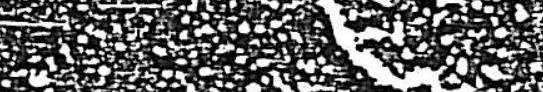

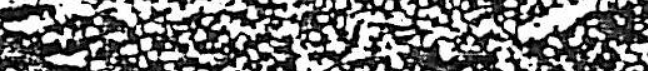

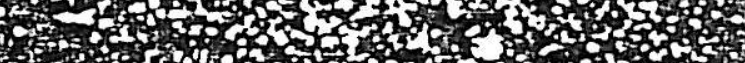
6. 40 to

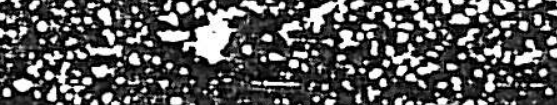

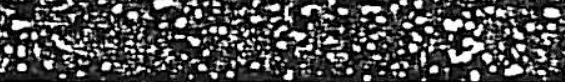

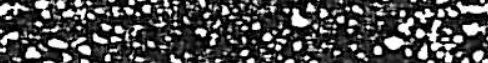


Virila
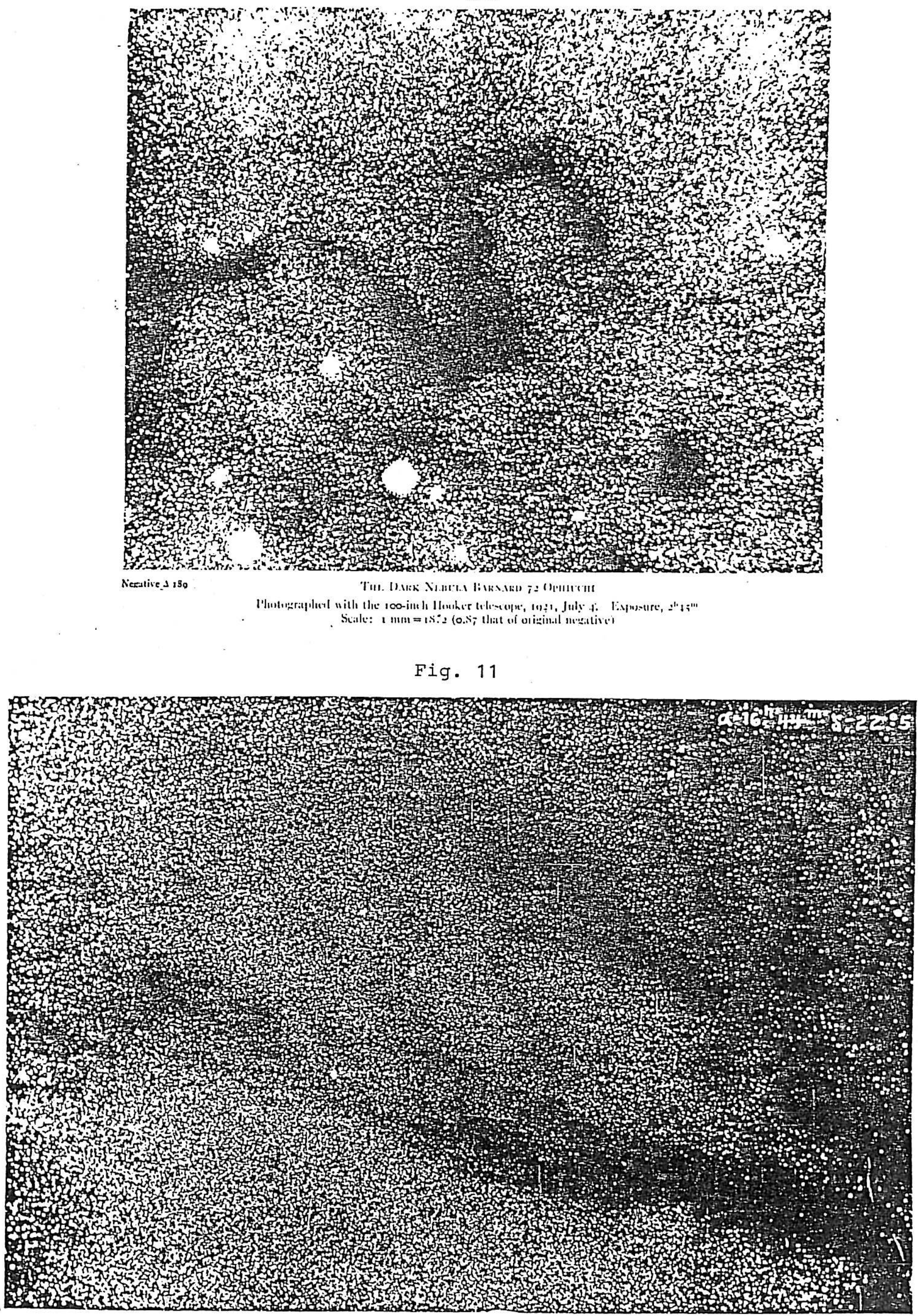


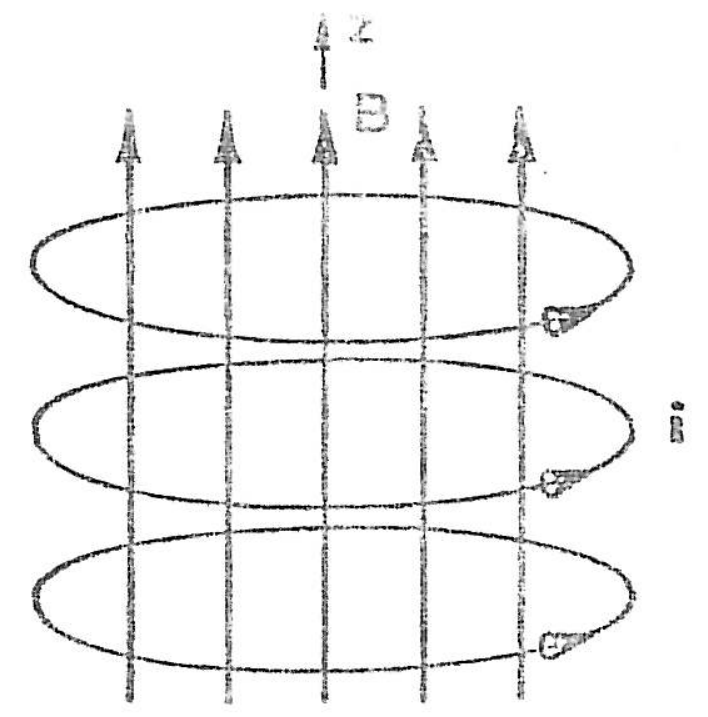

E.

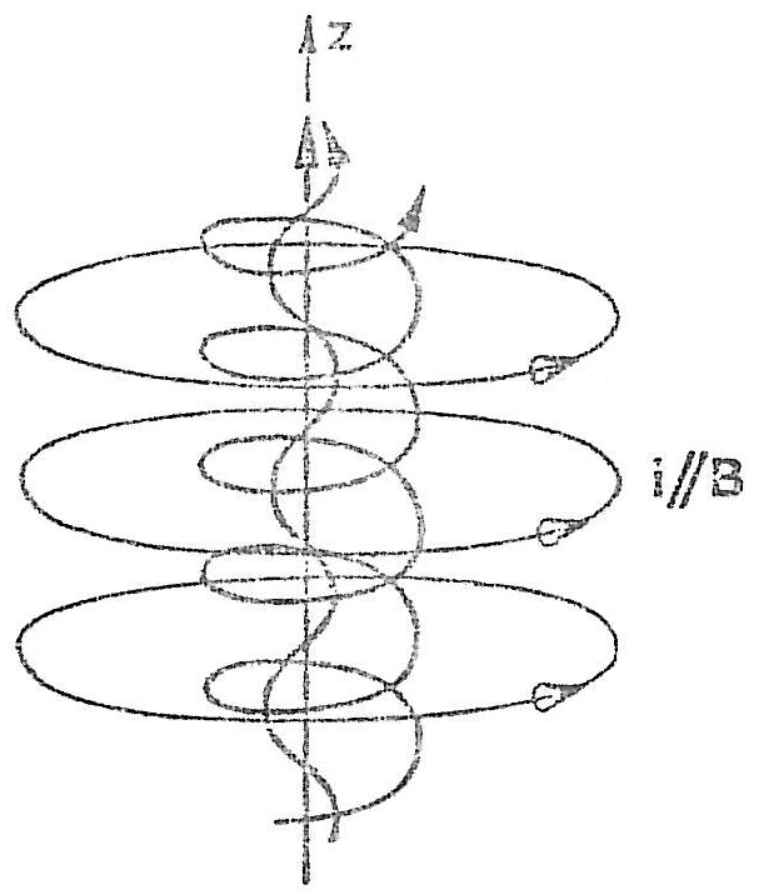

b.

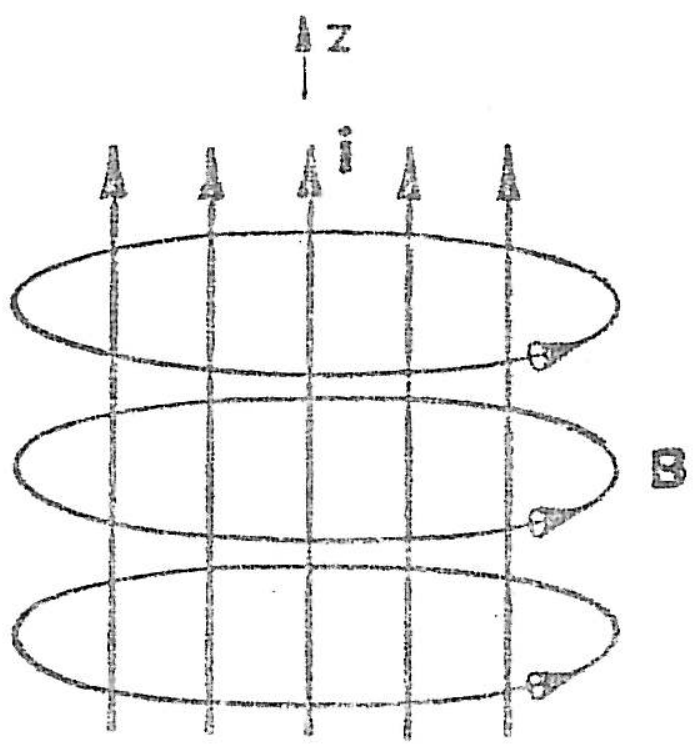

c.

Fig. 13 


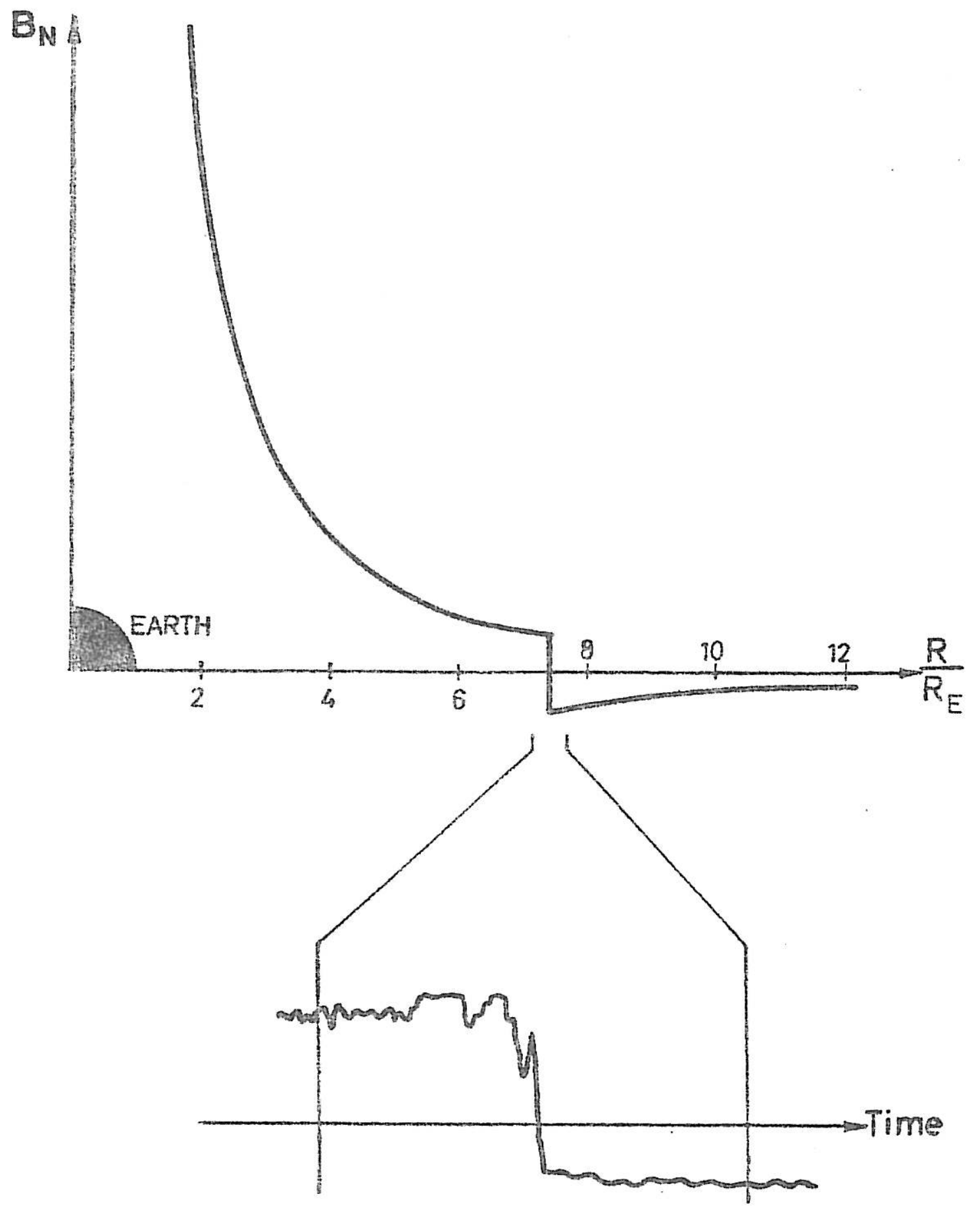

ISEE-l satellite data 10 nov 1977.

Fig. 14 


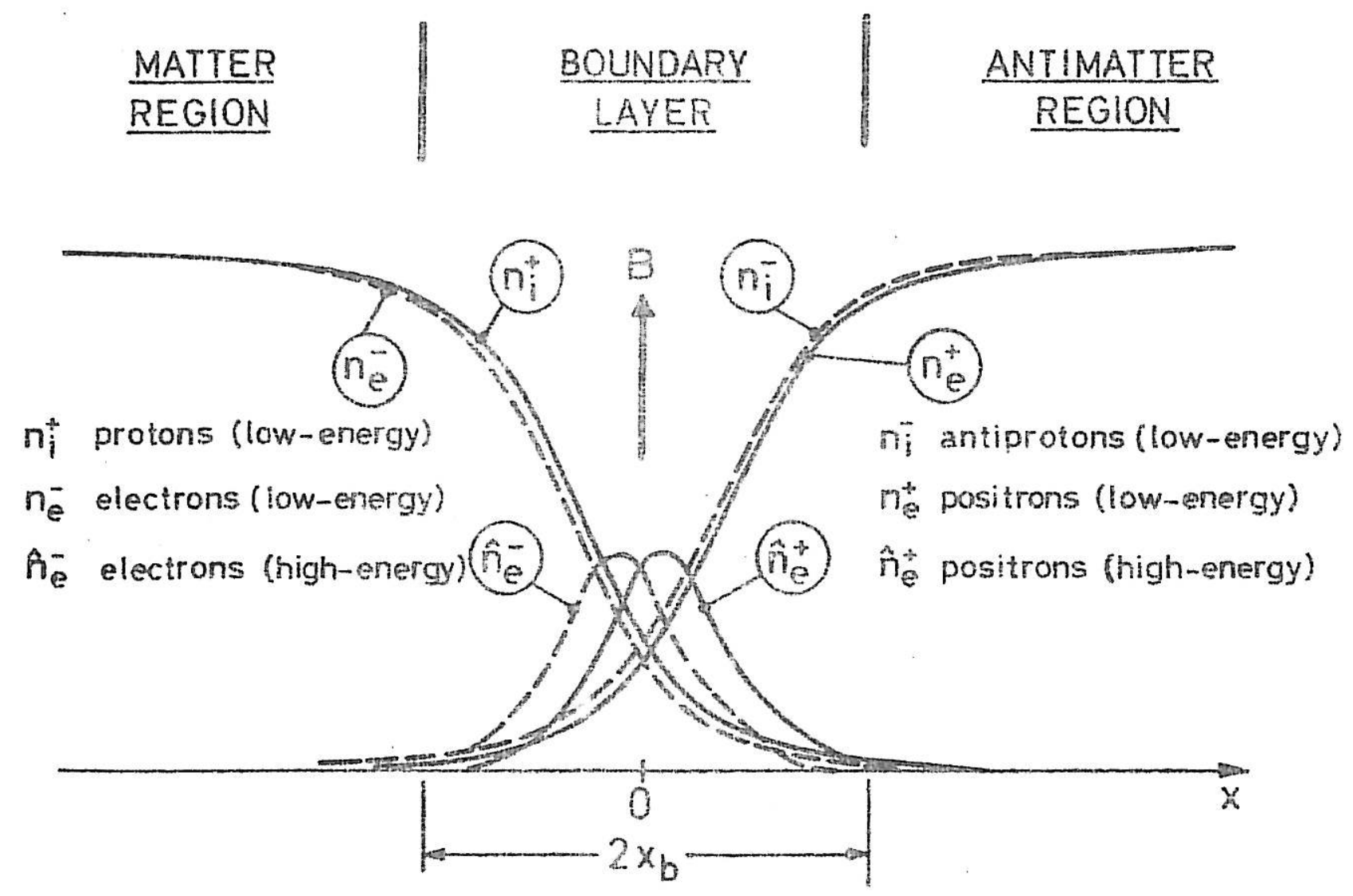

Fig. 15 
Royal Institute of Technology, Department of Plasma Physics, S-100 $\$ 4$ Stockholm, Sweden

PARADIGM TRANSIIION IN COSMIC PIASMA PHYSICS

\section{H. A.Ifvên}

June 1982, 40 pp. incl. ili., in English

In situ measurements in the magnetospheres together with general advancement in plasma physics are now necessitating introduction of a number of effects that have been recently discovered or earlier neglected. Eximpjes are:

Electric double layers (like in the lower magnetosphere) Thin current layer (like in the magnetopause) giving space a cellular structure.

Current produced filaments (e.g. in prominences, solar corona and interstellar clouds).

Further it is important to use the electric current (particle) description and to study the whole circuit in which the current flows. The pinch effect cannot be neglected as is now usually done. The critical velocity phenomenon is essential, for example for the band structure of solar system.

Theory of dusty plasmas is important.

The result is a change in so many theories in cosmic plasma physics that it is appropriate to speak of an introduction of a new paradigm. This should be based on empirical knowledge from magnetospheric and laboratory investigations. Its application to astrophysics in general, including cosmology, will necessarily lead to a revision of $e . g$. the present theories of the formation of stars, planets and satelitites. It is doubtiul whether the big bang cosmology will survive.

Key words: Plasma physics, Astrophystcs, Dowble layers, Electric circuits, Magnetopause, Pinch effect, Critical velocity, Dusty plasma, Origin of solar system, Cosmology. 